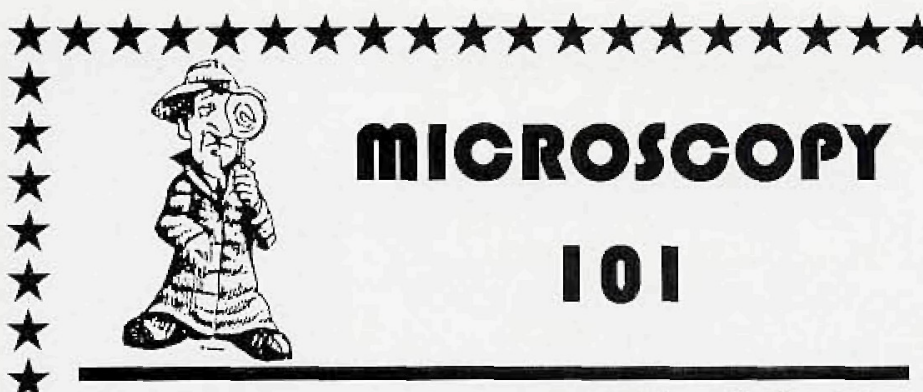

Thanks to Phil Oshel for his work in extending this publication feature. Our objective is to publish "hints" in all areas of microscopy which will aid readers in their professions. Contributions would be greatly appreciated and may be sent to Phil by:

eMail: oshel@ux1.cso.uiuc.edu

Snail Mail: Station A

PO Box 5037

Champaign /L 61825-5037

\section{Double-Sided Double-Labelling of Grids with Antibodies:}

Here is a nice solution to the problem of conjugated gold-antibody double-labeling, when both antibodies are monoclonals from the same animal (say, mouse monoclonals).

1. On one side of the grid (use fairly high hexagonal mesh, uncoated nickel grids) incubate with one of your mouse monoclonals

2. Follow with biotinylated goat anti-mouse, $\lg$-whatever, to match your primary antibody.

3. Follow with streptavidin-gold (available in many different sizes).

4. Flip the grid over and repeat the labeling with the second monoclonal, this time using a different size of streptavidin-gold. (Make certain that you have a clean surface for the grid! You'll be putting your sections directly on it, without using support films.)

5. Double-sided labeling works quite nicely for co-localizations, and it eliminates a lot of cross-reactivity problems you'd have to fight if you worked on only one surface of the section.

6. One thing to watch out for: If you add normal goat serum and maybe a little TWEEN or other additives to the reagents, the grids will tend to sink if they're incubated on drops of the reagents. Then you're labeling both sides with the same reagents. This, of course, is to be avoided! You have to keep the sides separated.

The best way to avoid this problem is to anchor the grid by its edge on a piece of double-stick tape on a microscope slide. You can then use a micropipette to deliver very small volumes (maybe 2-3 microliters) of each reagent. To change reagents or to wash, simply add the reagent to one edge of the grid and wick it off with filter paper from the opposite edge. Following the first gold incubation you can remove the grid and wash well in a gentle stream, or with repeated drops of buffer, and finally water. Allow to dry, flip the grid over, stick it down to the tape and do the second run-through.

An added bonus of this procedure is a fairly good signal amplification. If you conjugated your primary directly to the gold, you'd only have a one-step reaction with minimal gold particles (probably 1) binding at each site. That might not be enough to detect above background.

Unless, of course, you want the most specific localization possible - in that case, forget everything I just wrote! For highly specific localizations, you would probably want to opt for less amplification. In this case direct antibody conjugations to gold are certainly a possibility; you might also investigate eliminating the biotinylated secondary antibody and replacing this step with a protein A-gold conjugate. These techniques also work well with double-sided labeling.

Bob Chiovetti, Cutting Edge Consultants

\section{Micro Salt Plate Holder \\ for the FT-IR Microscope}

In the reference work PRACTICAL GUIDE TO INFRARED MICROSPECTROSCOPY by $\mathrm{H}$. Humecki, there is an excellent chapter (Chapter 11) by Anna Teetsov on preparation techniques for nanogram samples. On page 425 a slide holder made from aluminum is described for holding micro salt plates for the FT-IR microscope. I have made something similar for our lab using cardboard from a memo pad. Basically a 1 inch $\times 3$ inch piece can be cut with small holes punched near the center, across which the micro salt plates can be layed. I then coat the upper surface with rubber cement, such as Sanford's Rubber Cement, No. 00494 to give it a slightly tacky surface to hold the plates. Micro salt plates can be made from broken or cracked salt plates by cleaving them with a razor blade. Another source is to purchase random cut $\mathrm{KBr}$ crystals from Perkin-Elmer, or some other supplier. These random cut pieces can then be used "as is," or cleaved further. This provides a very low cost method of running micro samples on infra-red microscopes.

James Benko, Microspec Analytical

\section{Quantitative C Analysis by EDS}

Quantitative (even standardized) EDS analysis for carbon in $\mathrm{Nb}$ is difficult to impossible. The same applies for carbon in $\mathrm{Zr}$. $\mathrm{ZrC}$ can be even more difficult because (according to our metallurgists and ceramicists) it is not necessarily a stoichiometric compound. In addition, this makes locating a suitable standard difficult. (The following comments refer only to $\mathrm{Nb}$, but apply to $\mathrm{Zr}$ as well, and probably to other elements.)

There are several problems: a) very light element (C), theretbre soft $\mathrm{x}$-rays in a high $\mathrm{Z}$ matrix, b) $\mathrm{Nb}$ is a strong absorber of carbon x-rays, c) since the matrix carbon signal is weak, any surlace carbon (contamination) can be a major contributor to the carbon peak. Together, these make for very large absorption correction coefficients and magnify surface contamination contributions.

To maximize your chances, first don't rely on standardless (semi) quantitative analysis and second, a clean, high vacuum is required to minimize surface contamination (largely carbon) during analysis. Also, specimen surface preparation is crucial. A flat, well polished, and very clean specimen surface is essential. Finally, do not coat the specimen, as $\mathrm{NbC}$ is sufficiently conductive.

It is important to minimize analysis volume and depth. The best ways to do this are: a) use as low a beam voltage as practical, (recall that beam $\mathrm{kV}$ should be about 2.5 times the energy of the $x$-ray peak wanted), and b) high tilt angles will skew the volume to near the surface, therefore more $C x$-rays will escape to be detected (if the surface is facing the detector). There are two major caveats: first, the Nb L-line (family) response is less predictable than the $k \propto$ line response (I have run across some quantitative software which won't use L-lines, at least not for standardless quantitative analysis), and second, less-than-perfect quantitation compensation for high specimen tilt angles may add some error, especially for standardless analysis. (For standardless quantitative analysis, I have seen demos produce errors of over 300 percent.) References to look for include:

"Quantitative Electron Probe Microanalysis of Carbon in Binary Carbides" I "Principles and Procedures", X-ray Spectrometry 15:135-141,1986 II "Data Reduction and Comparison of Programs" Xray Spectrometry 15,1986 both by G. F. Bastin and H. J. M. Heijligers

N.W. White, Jr. (Woody),

Lynchburg Research Center, Babcock \& Wilcox Co.

\section{Stretching the Life of an Ion Pump}

lon pumps are used to evacuate the specimen and electron gun chambers of most of the high-resolution transmission electron microscopes that have been manufactured since the late 1970s. Many of these pumps have now been in service for a significant number of years, and some of them are developing instabilities which seriously degrade their pumping abilities. These instabilities 